\title{
Sostenibilidad ambiental del riego con agua marina desalinizada y reutilización de drenajes en tomate bajo invernadero
}

\section{B. Martin-Gorriz' ${ }^{\text {, J. F. Francisco Maestre-Valero }{ }^{1} \text {, B. Gallego-Elvira }{ }^{1} \text {, V. Martínez-Alvarez }}{ }^{1}$}

1 Escuela Técnica Superior de Ingeniería Agronómica, Universidad Politécnica de Cartagena. Paseo Alfonso XIII, 48. 30203 Cartagena, Spain; e-mail: b.martin@upct.es

\begin{abstract}
Resumen: La incorporación de agua marina desalinizada (AMD) al sureste español es la principal estrategia recogida en la planificación hídrica española con el fin de hacer frente a su déficit estructural de agua, que afecta principalmente al regadío. Este trabajo analiza la sostenibilidad medioambiental del riego con AMD en sistemas hidropónicos con reutilización de drenajes para el cultivo de tomate bajo invernadero. La metodología aplicada se basa en el Análisis de Ciclo de Vida (ACV) de acuerdo con la normativa ISO 14040. Esta metodología atribuye al producto final todos los efectos ambientales derivados del consumo de materias primas y de energías necesarias para su manufactura, las emisiones y residuos generados en el proceso de producción, así como los efectos ambientales procedentes del fin de vida del producto cuando éste se consume o ya no se puede utilizar. La metodología ha sido aplicada con los datos experimentales obtenidos mediante ensayos en el marco del proyecto LIFE-DESEACROP en la Fundación Finca Experimental UAL-ANECOOP, Retamar (Almería) y analiza específicamente y de forma comparativa los efectos de (1) las fuentes de agua convencionales frente al riego con AMD, y (2) los sistemas con reutilización de drenajes frente a los sistemas que vierten los drenajes al subsuelo. Los impactos ambientales analizados han sido el potencial de calentamiento global, el potencial de acidificación del suelo y el agua, el potencial de eutrofización, y el potencial de agotamiento de recursos abióticos. También se han incluido en el análisis dos indicadores de especial relevancia en la producción agrícola como la eficiencia del uso del agua y la demanda de energía acumulada. Como conclusiones del trabajo cabe destacar que, (1) el empleo de agua AMD supone un aumento leve del impacto ambiental en la mayoría de categorías de impacto analizadas ( $<5 \%)$; (2) el cultivo hidropónico tiene una mayor eficiencia del uso del agua que el cultivo en suelo (14\%) y (3) la reutilización de drenajes reduce la acidificación, eutrofización y agotamiento de recursos, pero aumenta el calentamiento global y la demanda de energía acumulada.
\end{abstract}

Palabras clave: Análisis ciclo vida; sostenibilidad ambiental; emisiones de efecto invernadero; aguas no convencionales.

\section{Introducción}

La agricultura, además de ser competitiva para producir alimentos de alta calidad, debe ser sostenible [1, 2]. Para mejorar la sostenibilidad se requiere un mayor conocimiento sobre los impactos ambientales de las actividades agrícolas habituales. El análisis del ciclo de vida (ACV), es una metodología ampliamente aceptada para evaluar las cargas ambientales asociadas a un producto, proceso o actividad y, en base a ellas diagnosticar su sostenibilidad ambiental. Además, permite evaluar y llevar a la práctica estrategias de mitigación. Diversos autores han utilizado esta 


\section{CONGRESO IBÉRICO DE AGROINGENIERÍA \\ X CONGRESSO IBÉRICO DE AGROENGENHARIA \\ 3 - 6 septiembre 2019, Huesca - España}

metodología para comparar diferentes sistemas de producción de tomate bajo invernadero $[3,4,5$, 6]. Algunos de ellos se han centrado exclusivamente en determinar la huella de carbono en el cultivo hidropónico de tomate [4, 7], y muy pocos [8], en comparar la sostenibilidad del cultivo de tomate entre sistemas con sustrato y con recirculación de drenaje; por lo que la novedad que presenta este trabajo es la valoración conjunta del efecto del uso de agua desalinizada y la reutilización del drenaje en la sostenibilidad del cultivo de tomate en invernadero.

\section{Materiales y métodos}

Este trabajo analiza la sostenibilidad medioambiental del riego con AMD (agua marina desalinizada) en sistemas hidropónicos con reutilización de drenajes para el cultivo de tomate bajo invernadero, a partir de los datos experimentales obtenidos en un ensayo realizado en la Fundación Finca Experimental UAL-ANECOOP, Retamar (Almería).

En el estudio se analiza específicamente y de forma comparativa los efectos de (1) las fuentes de agua convencionales frente al riego con AMD, y (2) los sistemas con reutilización de drenajes frente a los sistemas que vierten los drenajes al subsuelo. Para cuantificar el punto (1), i.e. las diferencias entre el uso de AMD y aguas salobres disponibles en la zona de estudio, se realizaron tres tratamientos con diferente salinidad: uno con agua desalinizada suministrada por la planta desaladora de Carboneras (T1), y otros dos con aguas de mayor conductividad (T2 y T3) obtenidos por mezcla de AMD y agua salobre de pozo. Para cuantificar el punto (2), i.e. el impacto de la recirculación de drenajes frente a sistemas abiertos, se instaló un módulo de osmosis inversa alimentado por placas solares que permitió reutilizar parcialmente el agua de los drenajes.

Los ensayos se llevaron a cabo en un invernadero de raspa y amagado tipo Almería sin calefacción y con ventilación cenital natural automatizada. Se utilizaron dos sistemas de cultivo: enarenado tradicional de la zona como sistema sin reutilización de drenajes; y cultivo hidropónico en fibra de coco con sistema con reutilización de drenajes. Los resultados que se muestran en este estudio corresponden al cultivo con plantas de tomate (Solanum lycopersicum L. cv. Ramyle) realizado durante un ciclo otoño-primavera (2018-2019).

\subsection{Analisis de ciclo de vida}

Para el análisis se ha utilizado la metodología de ACV de acuerdo con la normativa ISO 14040. El ACV es un proceso objetivo que permite evaluar las cargas ambientales asociadas a un producto o cultivo agrícola, identificando y cuantificando el uso de materia y energía y los vertidos al entorno, con el fin de determinar el impacto que ese uso de recursos y esos vertidos producen al medio ambiente. Además, permite evaluar y llevar a la práctica estrategias de mejora ambiental, así como brindar soporte para la toma de decisiones más rentables, sostenibles y eficientes. Para ello es necesario cuantificar los consumos de materias primas y energía junto con todos los residuos sólidos, emisiones a la atmósfera y vertidos al agua derivados de cada uno de los dos sistemas de producción estudiados. En el caso de los sistemas agrícolas la principal función es la producción de alimentos [9], por tanto, se ha considerado como unidad funcional (FU) de referencia la producción comercial de tomate (1 kg de peso fresco comercial).

\subsection{Categorias de impacto}

Las categorías de impacto analizadas (Tabla 1) han sido seleccionadas por su relevancia en los procesos agrícolas. Las emisiones relacionadas con procesos agrícolas, las infraestructuras y los fertilizantes son los principales contribuidores al cambio climático. Las emisiones de amonio y nitratos debido al empleo de fertilizantes nitrogenados contribuyen de forma significativa a las categorías eutrofización y acidificación. El agotamiento de recursos abióticos y energéticos naturales se encuentra relacionado con la extracción de minerales y combustibles fósiles, que se producen en la fabricación de los materiales empleados en los sistemas de producción agrícola. 


\section{CONGRESO IBÉRICO DE AGROINGENIERÍA \\ X CONGRESSO IBÉRICO DE AGROENGENHARIA \\ 3 - 6 septiembre 2019, Huesca - España}

Por último, se ha considerado la eficiencia del uso del agua, que es un buen indicador del aprovechamiento de los recursos hídricos. Para la evaluación de las categorías de impacto (Tabla 1) se ha utilizado la metodología CML-IA [10] con el software SimaPro 8.5 [11].

Tabla 1. Categorías de impacto consideradas en el análisis.

\begin{tabular}{ccc}
\hline Categoría de impacto & Acrónimo & Unidad \\
\hline Agotamiento de recursos & $\mathrm{ADP}$ & $\mathrm{kg} \mathrm{Sb}$ eq $/ \mathrm{FU}^{*}$ \\
Calentamiento global & $\mathrm{GWP}$ & $\mathrm{kg} \mathrm{CO} 2 \mathrm{eq} / \mathrm{FU}$ \\
Acidificación & $\mathrm{AP}$ & $\mathrm{kg} \mathrm{SO} 2 \mathrm{eq} / \mathrm{FU}$ \\
Eutrofización & $\mathrm{EP}$ & $\mathrm{kg} \mathrm{PO}-3$ eq/FU \\
Demanda de energía & $\mathrm{CED}$ & $\mathrm{MJ} / \mathrm{FU}$ \\
Eficiencia del uso del agua & $\mathrm{WU}$ & $\mathrm{FU} / \mathrm{m}^{3}$ \\
\hline
\end{tabular}

*UF: Unidad Funcional.

\subsection{Inventario de entradas-salidas}

El inventario del Ciclo de Vida permite cuantificar los consumos de materias primas y energía junto con todos los residuos sólidos, emisiones a la atmósfera y vertidos al agua (cargas medioambientales) derivados de todos los procesos que están dentro de los sistemas de producción del tomate considerados. En la Tabla 2 se muestra el inventario de entradas y salidas del ensayo para cada uno de los sistemas de cultivo.

Tabla 2. Inventario de entradas y salidas en los sistemas de cultivo analizados.

\begin{tabular}{|c|c|c|c|}
\hline \multirow[t]{2}{*}{ Entradas / salidas } & \multirow{2}{*}{$\begin{array}{l}\text { Unidades } \\
\text { por ciclo }\end{array}$} & \multicolumn{2}{|c|}{ Sistema de cultivo } \\
\hline & & $\begin{array}{l}\text { Sin recirculación del } \\
\text { drenaje (enarenado) }\end{array}$ & $\begin{array}{c}\text { Con recirculación del } \\
\text { drenaje } \\
\text { (fibra de coco) }\end{array}$ \\
\hline Agua para riego & $\mathrm{L} / \mathrm{m} 2$ & 278 & 278 \\
\hline Drenaje (estimado) & $\%$ & $5 \%$ & - \\
\hline Lixiviado & $\%$ & - & $20 \%$ \\
\hline \multicolumn{4}{|c|}{ Consumo especifico por fuente de agua: } \\
\hline - T1: agua desalada (1 ds/m) & $\mathrm{kWh} / \mathrm{m} 3$ & 4.98 & 4.98 \\
\hline - T2 agua mezcla (2 ds/m) & $\mathrm{kWh} / \mathrm{m} 3$ & 3.69 & 3.69 \\
\hline - T3: agua mezcla (3 ds/m) & $\mathrm{kWh} / \mathrm{m} 3$ & 1.77 & 1.77 \\
\hline \multicolumn{4}{|l|}{ Fertilizantes: } \\
\hline$-\mathrm{N}$ & $\mathrm{g} / \mathrm{m} 2$ & 260 & 260 \\
\hline - P2O5 & $\mathrm{g} / \mathrm{m} 2$ & 180 & 180 \\
\hline$-\mathrm{K} 2 \mathrm{O}$ & $\mathrm{g} / \mathrm{m} 2$ & 490 & 490 \\
\hline \multicolumn{4}{|l|}{ Pesticidas: } \\
\hline - Insecticidas & $\mathrm{g} / \mathrm{m} 2$ & 38 & 38 \\
\hline - Fungicidas & $\mathrm{g} / \mathrm{m} 2$ & 285 & 285 \\
\hline \multicolumn{4}{|l|}{ Sustratos: } \\
\hline - Estiércol para enarenado & $\mathrm{Kg} / \mathrm{m} 2$ & 2.53 & - \\
\hline - Fibra de coco & $\mathrm{Kg} / \mathrm{m} 2$ & - & 1.0 \\
\hline Producción & $\mathrm{Kg} / \mathrm{m} 2$ & 7.21 & 8.97 \\
\hline Duración ciclo & días & 163 & 163 \\
\hline Densidad de plantación & Plantas/m2 & 2 & 2 \\
\hline
\end{tabular}

Otros datos necesarios para el cálculo de las emisiones han sido obtenidos de la base de datos Ecoinvent 3.5. [12], incluyendo la fabricación de los materiales necesarios para la construcción del invernadero y material plástico utilizado en los sistemas de cultivo, y los materiales empleados en la instalación del equipo de riego, equipo de tratamiento de lixiviado, 


\section{CONGRESO IBÉRICO DE AGROINGENIERÍA \\ X CONGRESSO IBÉRICO DE AGROENGENHARIA \\ 3 - 6 septiembre 2019, Huesca - España}

etc., así como la fabricación de fertilizantes y pesticidas, producción de electricidad, transporte de materiales y residuos a planta de procesado. Por otra parte, las emisiones de N $\left(\mathrm{NH}_{3} ; \mathrm{N}_{2} \mathrm{O}\right.$; $\left.\mathrm{NO}_{3}\right)$ al aire y agua, y $\mathrm{P}\left(\mathrm{PO}_{4}\right)$ al agua durante la fase de producción del tomate fueron calculadas siguiendo la metodología propuesta en [13, 14].

Para poder realizar una evaluación ambiental detallada, el proceso de producción ha sido dividido en cuatro fases: (1) Estructura del invernadero, (2) Equipo auxiliar, (3) Fertilizantes y pesticidas y (4) Manejo del cultivo. En la Fig. 1 se muestra el diagrama de flujo para las fases consideradas en la producción de tomate.

En la estructura del invernadero se ha tenido en cuenta la fabricación y transporte del material necesario para crear la estructura y construcción del invernadero y material plástico de la cubierta, pared etc. Esta información ha sido obtenida de la base de datos Ecoinvent 3.5 para un invernadero de características similares al utilizado en el ensayo. En dicha base de datos se consideran las emisiones producidas en la fabricación de los materiales empleados, su extracción y transporte hasta el lugar de producción; así como la gestión de todos los residuos generados al final de la vida útil de la estructura, incluyendo su transporte hasta planta gestora.

En el equipo auxiliar se han considerado todos los materiales empleados en la fabricación de los elementos necesarios en el sistema de riego, así como el transporte de los mismos y la energía consumida por el funcionamiento del sistema de riego. Los materiales empleados en este proceso fueron: acero en las bombas de riego e inyectores; polietileno en tuberías, válvulas y depósitos, incluido el transporte (tipo de vehículo y $\mathrm{km}$ recorridos); y el consumo eléctrico asociado al uso del agua, tanto dentro del invernadero para realizar el riego, como fuera para producirla y/o transportarla. De igual manera que en la fase de estructura del invernadero, se ha considerado la gestión de los residuos al final de la vida útil.

En fertilizantes y pesticidas se ha incluido, para los fertilizantes, su proceso de fabricación, las cantidades totales de nitrógeno $(\mathrm{N})$, fósforo $\left(\mathrm{P}_{2} \mathrm{O}_{5}\right)$ y potasio $\left(\mathrm{K}_{2} \mathrm{O}\right)$ aplicadas durante el ciclo de cultivo, así como las emisiones de amoniaco $\left(\mathrm{NH}_{3}\right)$, monóxido de dinitrógeno $\left(\mathrm{N}_{2} \mathrm{O}\right)$ y óxidos de nitrógeno $\left(\mathrm{NO}_{\mathrm{x}}\right)$ al aire y lixiviación de nitratos $\left(\mathrm{NO}_{3}\right)$ y fosfatos $\left(\mathrm{PO}_{4}\right)$ al agua. En esta fase también se han considerado, para el sistema de reutilización de drenajes, la planta de osmosis para el tratamiento del lixiviado; con lo que se evita la contaminación de nitratos y fosfatos al subsuelo. En la planta de osmosis no se ha considerado el consumo de energía eléctrica puesto que disponía de paneles solares para su funcionamiento. Respecto a los pesticidas, se ha incluido su fabricación, transporte y el uso de maquinaria y energía consumida para su aplicación.

En manejo del cultivo, para ambos sistemas de cultivo se ha considerado el plástico del suelo, cuerdas, etc. En el cultivo enarenado se ha incluido el tractor y maquinaria empleada para realizar el enarenado, incluido el combustible; así como el estiércol y las emisiones producidas por el mismo [emisiones de amoniaco $\left(\mathrm{NH}_{3}\right)$ y monóxido de dinitrógeno $\left(\mathrm{N}_{2} \mathrm{O}\right)$ y óxidos de nitrógeno $\left(\mathrm{NO}_{x}\right)$ al aire y lixiviación de nitratos $\left(\mathrm{NO}_{3}\right)$ al agua]. En el cultivo hidropónico se incluyen los sacos de fibra de coco; su procesado y transporte, así como las canaletas y otros materiales plásticos específicos para el riego en hidropónico. Como residuos, para ambos tipos de sustrato, se han considerado el transporte y gestión de la biomasa ( $40 \%$ humedad) de las plantas al final del ciclo, enviadas al centro de compostaje, y los plásticos al centro de gestión. Para el cultivo hidropónico se ha tenido en cuenta también el transporte de la fibra de coco y plásticos (canaletas, etc.) al centro de gestión. 


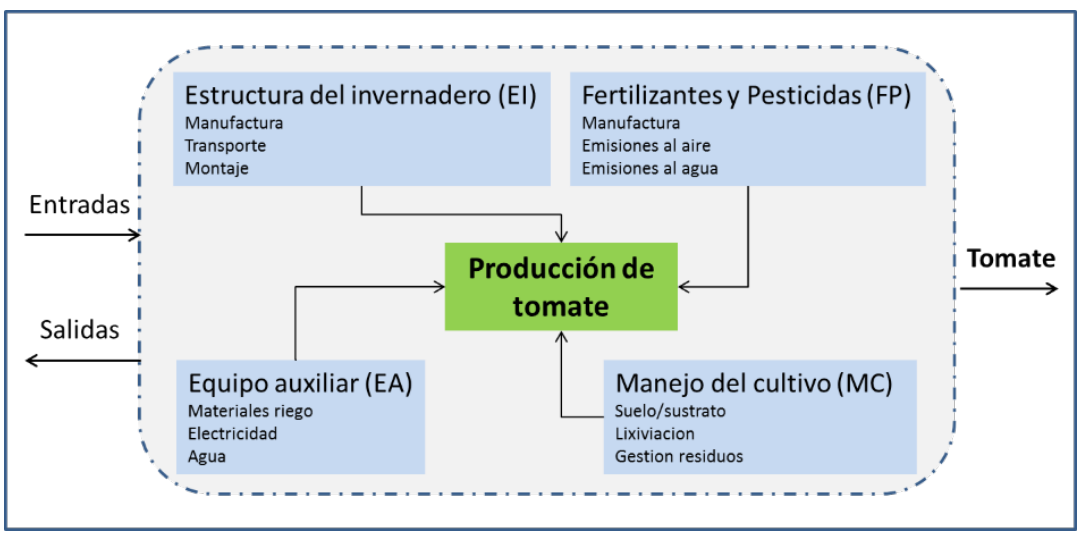

Figura 1. Diagrama de flujo para las fases consideradas en la producción de tomate.

\section{Resultados y discusión}

En la Tabla 3 se muestran los impactos ambientales de la producción de $1 \mathrm{~kg}$ de tomate en los dos sistemas de cultivo y tres tratamientos considerados. El uso de AMD frente a agua salobre, tanto en cultivo enarenado como en cultivo hidropónico, produjo un incremento leve del impacto ambiental en todas las categorías de impacto que se atribuyó principalmente a que el impacto asociado al volumen de agua empleado en el cultivo fue bajo en comparación con otros inputs. Incluso en los tratamientos que utilizaron mayor volumen de AMD (T1S y T1F frente a T3S y T3F), que conllevaron un mayor consumo de energía específica, los incrementos en todas las categorías de impacto fueron inferiores al 5\%, exceptuando la CED, donde se alcanzó el 10\% entre T1S y T3S. La intensificación de los sistemas agrícolas, como en el caso de los cultivos hidropónicos, incrementaron el requerimiento de energía fósil que repercutió directamente en las categorías de impacto relacionadas como CED y GWP $[15,16]$.

La reutilización de los drenajes en los sistemas de cultivo hidropónico redujo la AP, EP y $\mathrm{ADP}$ en un 28,27 y $20 \%$, respectivamente, frente a los sistema de cultivo en suelo, independientemente del tratamiento de riego; pero aumentó el impacto en GWP y CED en un 14 y 6\% respectivamente (Tabla 3). Nuestros resultados están en línea con los obtenidos por [8], quienes evaluaron el impacto ambiental de tres sistemas de cultivo de tomate en invernadero, $i$. $e$. cultivo en suelo y cultivo hidropónico en ciclo cerrado y abierto. Sin embargo, no es posible comparar directamente los valores obtenidos porque tanto la metodología utilizada como las fases del proceso fueron diferentes.

Tabla 3. Comparación de los impactos ambientales de la producción de $1 \mathrm{~kg}$ de tomate en los seis sistemas de cultivo considerados.

\begin{tabular}{cccccccc}
\hline $\begin{array}{c}\text { Categoría } \\
\text { impacto }\end{array}$ & Unidad & & & & & & \\
& & T1S & T1F & T2S & T2F & T3S & T3F \\
\hline $\mathrm{ADP}$ & $\mathrm{kg} \mathrm{Sb} / \mathrm{FU}$ & $1.07 \mathrm{E}-05$ & $8.60 \mathrm{E}-06$ & $1.06 \mathrm{E}-05$ & $8.48 \mathrm{E}-06$ & $1.03 \mathrm{E}-05$ & $8.31 \mathrm{E}-06$ \\
$\mathrm{GWP}$ & $\mathrm{kg} \mathrm{CO}$ eq/FU & 0.383 & 0.432 & 0.380 & 0.430 & 0.375 & 0.426 \\
$\mathrm{AP}$ & $\mathrm{kg} \mathrm{SO}_{2}$ eq $/ \mathrm{FU}$ & $2.16 \mathrm{E}-03$ & $1.58 \mathrm{E}-03$ & $2.16 \mathrm{E}-03$ & $1.58 \mathrm{E}-03$ & $2.16 \mathrm{E}-03$ & $1.58 \mathrm{E}-03$ \\
$\mathrm{EP}$ & $\mathrm{kg} \mathrm{PO}^{-3}$ eq $/ \mathrm{FU}$ & $8.71 \mathrm{E}-04$ & $6.34 \mathrm{E}-04$ & $8.55 \mathrm{E}-04$ & $6.21 \mathrm{E}-04$ & $8.30 \mathrm{E}-04$ & $6.02 \mathrm{E}-04$ \\
$\mathrm{CED}$ & $\mathrm{MJ} / \mathrm{FU}$ & 7.65 & 7.94 & 7.38 & 7.72 & 6.96 & 7.38 \\
$\mathrm{WU}$ & $\mathrm{FU} / \mathrm{m}^{3}$ & 25.6 & 37.0 & 25.6 & 37.0 & 25.6 & 37.0 \\
\hline
\end{tabular}

T1S: cultivo en enarenado regado con agua desalada; T1F: cultivo en fibra de coco regado con agua marina desalada; T2S: cultivo en enarenado regado con agua de pozo; T2F: cultivo en fibra de coco regado con agua de pozo; T3S: cultivo en enarenado regado con agua mezcla; T3F: cultivo en fibra de coco regado con agua mezcla. 


\section{CONGRESO IBÉRICO DE AGROINGENIERÍA \\ X CONGRESSO IBÉRICO DE AGROENGENHARIA \\ 3 - 6 septiembre 2019, Huesca - España}

WU fue mayor en los sistemas de cultivo hidropónico que en los sistemas de enarenado ( 37.0 frente a $25.6 \mathrm{~kg} / \mathrm{m}^{3}$ ), debido a la recuperación del $20 \%$ del lixiviado (Tabla 3). Este lixiviado fue tratado en la planta de osmosis, con un rechazo medio de un $30 \%$, lo que supuso un ahorro de agua del $14 \%$. Este resultado coincide con el obtenido por otros autores como [16], que indicaron una mayor eficiencia del uso del agua en el cultivo hidropónico de lechuga frente al cultivo en suelo.

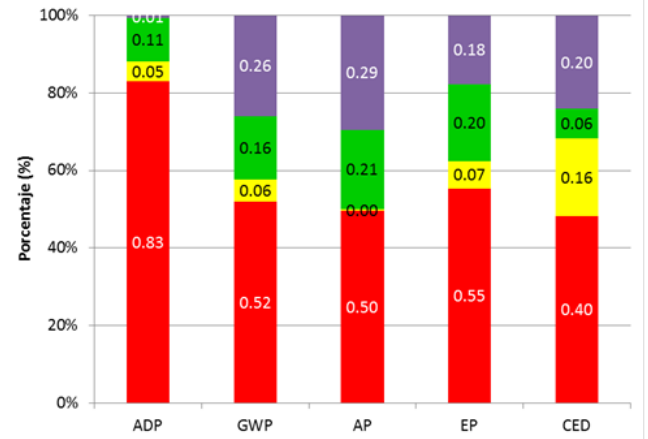

a) Cultivo en enarenado regado con agua T1.

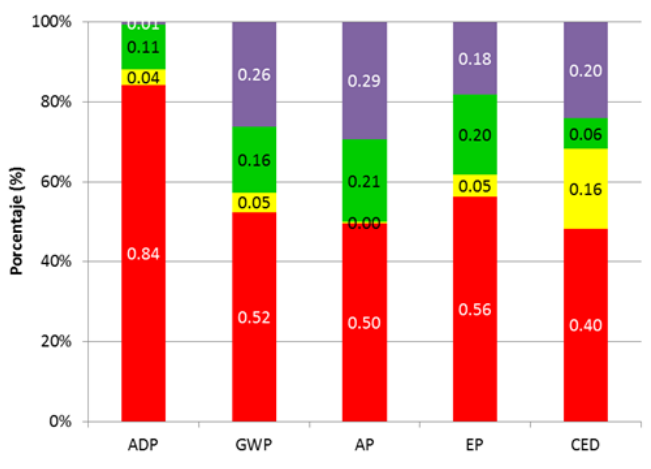

c) Cultivo en enarenado regado con agua T2.

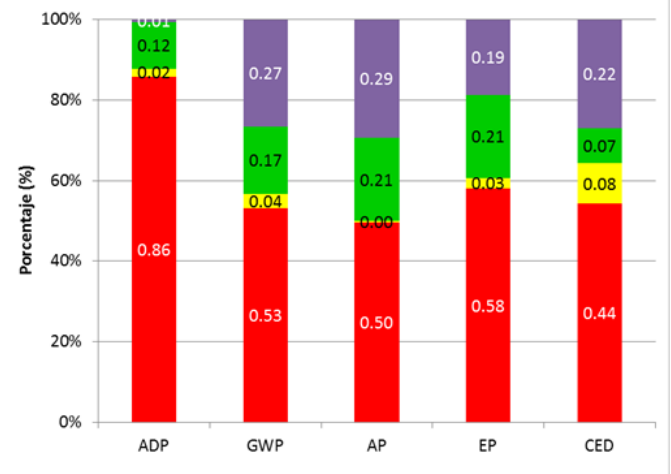

e) Cultivo en enarenado regado con agua T3.

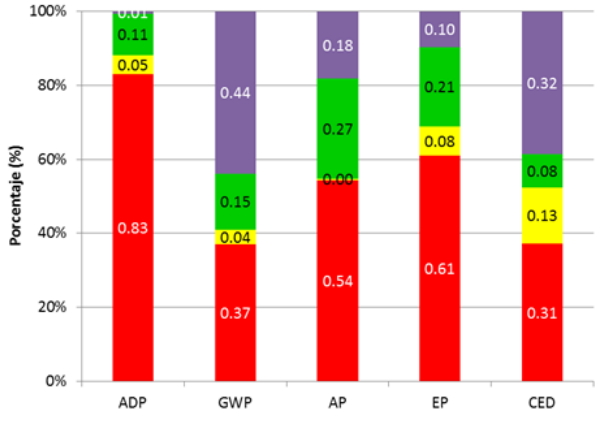

b) Cultivo en hidropónico regado con agua T1.

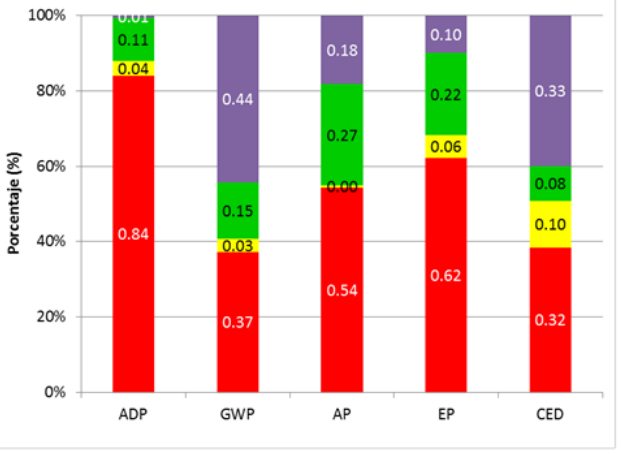

d) Cultivo en hidropónico regado con agua T2.

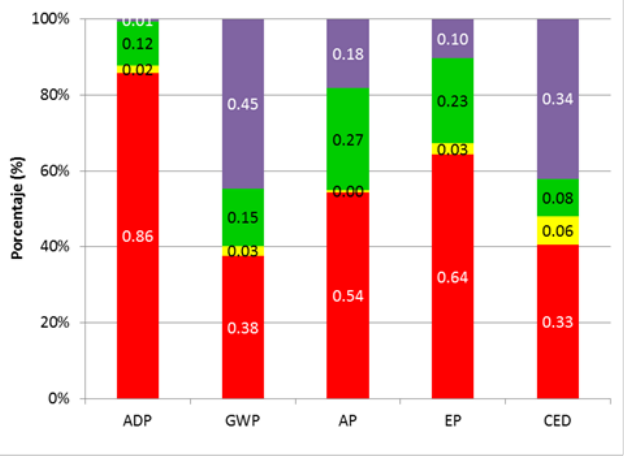

f) Cultivo en hidropónico regado con agua T3.

Figura 2. Contribución ambiental de cada fase (estructura del invernadero: barras rojas; equipamiento auxiliar: barras amarillas; fertilizantes y pesticidas: barras verdes; manejo del cultivo: barras lilas) para las categorías de impacto consideradas (ADP: agotamiento de recursos; GWP: calentamiento global, AP: acidificación, EP: eutrofización; CED: demanda de energía) en las 6 estrategias de producción de tomate bajo invernadero. T1: agua procedente de desaladora; T2 y T3 mezclas de agua de desaladora y agua de pozo. 


\section{CONGRESO IBÉRICO DE AGROINGENIERÍA \\ X CONGRESSO IBÉRICO DE AGROENGENHARIA \\ 3 - 6 septiembre 2019, Huesca - España}

La Fig. 2 muestra la contribución de cada fase del proceso en las categorías de impacto seleccionadas, para cada uno de los seis sistemas de producción de tomate bajo invernadero considerados. La estructura de invernadero (barras rojas en Fig. 2) es la fase con mayor carga ambiental en la mayoría de categorías. Destaca con mayor relevancia en la categoría de ADP debido a la alta cantidad de materiales empleados, como acero en la estructura y plástico en la cubierta. Por otra parte, la fase de manejo del cultivo (barras lilas en Fig. 2) comparativamente tiene mayor importancia en las categorías de impacto GWP y CED en los sistemas de cultivo hidropónico que en los sistemas de cultivo en suelo (sistemas $b, d$ y $f$ frente a sistemas $a, c$ y $e$ en Fig. 2); siendo el $44 \%$ en GWP y el 32\% en CED en los sistemas de cultivo hidropónico, mientras que en los sistemas de cultivo en suelo fue del 27 (GWP) y 20\% (CED). Esto se debió principalmente al elevado consumo de energía en la fabricación de los plásticos utilizados y al procesado y transporte de la fibra de coco. Sin embargo, en los sistemas de cultivo hidropónico $(b, d$ y $f$ en Fig. 2) se redujo el impacto ambiental frente al cultivo en suelo en las categorías AP y $\mathrm{EP}$, que pasaron de $\mathrm{AP}=29 \%$ y $\mathrm{EP}=18 \%$ a $\mathrm{AP}=18 \%$ y $\mathrm{EP}=10 \%$ como consecuencia de la recuperación del lixiviado de $\mathrm{NO}_{3}$ y $\mathrm{PO}_{4}$ del agua de riego.

\section{Conclusiones}

Las principales conclusiones del presente trabajo son:

- El empleo de AMD supone un aumento leve (inferior al 5\%) del impacto ambiental en la mayoría de categorías de impacto analizadas.

- La reutilización de drenajes reduce la acidificación, eutrofización y agotamiento de recursos, pero aumenta el calentamiento global y demanda de energía acumulada.

- El cultivo hidropónico con reutilización de drenajes permite incrementar en un $44 \%$ la eficiencia en el uso del agua con respecto al cultivo en suelo.

\section{Agradecimientos}

Trabajo financiado por el Programa LIFE de la Unión Europea a través del proyecto DESEACROP (LIFE16-ENV-ES-000341).

\section{Referencias}

1. CEC (Commission of the European Communities). Directions towards sustainable agriculture. 1999. Commission of the European Communities, Bruselas, Países Bajos.

2. UN-DSD (United Nations Division for Sustainable Development). 2000. Agenda 21. Chapter 14: Promoting sustainable agriculture and rural development. United Nations (UN), Nueva York, Estados Unidos.

3. Cellura M., Longo S., Mistretta M. Life cycle assessment (LCA) of protected crops: an Italian case study. 2012. J. Clean. Prod. 28, 56-62.

4. Ntinas G., Neumair M., Tsadilas C., Meyer J. Carbon footprint and cumulative energy demand of greenhouse and open-field tomato cultivation systems under Southern and Central European climatic conditions. 2017. J. Clean. Prod. 142, 3617-3626.

5. Perez D., Soler M., Delgado M., Reigada A. Energy use and carbon footprint of the tomato production in heated multi-tunnel greenhouses in Almeria within an exporting agri-food system context. 2018. Sci. Total Environ. 2018, 628, 1627-1636.

6. Torrellas M., Antón A., López J.C., Baeza E.J., Pérez-Parra J., Muñoz P., Montero J.I. LCA of a tomato crop in a multi-tunnel greenhouse in Almería. Int. J. Life Cycle Assess. 2012, 17, 863-875.

7. Page G., Ridoutt B., Bellotti B. Carbon and water footprint tradeoffs in fresh tomato production. 2012. J. Clean. Prod. 32, 219-226.

8. Antón A., Montero J.I., Muñoz P., Castells F. LCA and tomato production in Mediterranean greenhouses, Int. J. Agric. Resour. Gov. Ecol. 2005, 4 (2), 102-112.

9. Audsley E., Alber S., Clift R., Cowell S., Crettaz P., Gaillard G., Hausheer J., Jolliet O., Kleijn R., Mortensen B., Pearce D., Roger E., Teulon H., Weidema B., van Zeijts H. 1997. Harmonisation of 


\section{CONGRESO IBÉRICO DE AGROINGENIERÍA \\ X CONGRESSO IBÉRICO DE AGROENGENHARIA \\ 3 - 6 septiembre 2019, Huesca - España}

environmental life cycle assessment for agriculture. Final report concerted action AIR3- CT94-2028. European Commission DG VI Agriculture, Reino Unido.

10. Guinée J.B., Gorrée M., Heijungs R., Huppes G., Kleijn R., de Koning A., van Oers L., Wegener Sleeswijk A., Suh S., Udo de Haes H.A., de Bruijn H., van Duin R., Huijbregts M.A.J. 2002. Handbook on life cycle assessment. Operational guide to the ISO standards. Part III: Scientific background. Kluwer Academic Publishers, ISBN 1-4020-0228-9, Dordrecht, 692 p.

11. PRe Consultants, 2018 [www.pre-sustainability.com]

12. Ecoinvent, 2018. Ecoinvent Data. Swiss Centre for Life Cycle Inventories [http://www.ecoinvent.org]

13. Audsley E. Systematic procedures for calculating agricultural performance data for comparing systems. 2000. En: Weidema, B., Meeusen, M. (eds.). Agricultural data for life cycle assessments, 1, 35-46. Agricultural Economics Research Institute, La Haya, Países Bajos.

14. Brentrup F., Küsters J., Lammel J., Kuhlmann H. Methods to estimate on-field nitrogen emissions from crop production as an input to LCA studies in the agricultural sector. Int. J. Life Cycle Assess. 2000, 5(6), 349-357.

15. Castoldi N., Bechini L., Ferrante A. Fossil energy usage for the production of baby leaves. Energy, 2011, 36, 86-93.

16. Martinez-Mate M.A., Martin-Gorriz B., Martínez-Alvarez V., Soto-García M., Maestre-Valero J.F. Hydroponic system and desalinated seawater as an alternative farm-productive proposal in water scarcity areas: energy and greenhouse gas emissions analysis of lettuce production in southeast Spain. J. Clean. Prod. 2018, 172, 1298-1310. 